Original Research

\title{
A Neural Network Evidence of the Nexus Among Air Pollution, Economic Growth, and COVID-19 Deaths in the Hubei Area
}

\author{
Cosimo Magazzino, Marco Mele
}

Department of Political Sciences, Roma Tre University; E-Mails: cosimo.magazzino@uniroma3.it; marco.mele@uniroma3.it

* Correspondence: Cosimo Magazzino; E-Mail: cosimo.magazzino@uniroma3.it

Academic Editor: Dimitris Kaskaoutis

Special Issue: Urban Air Pollution

Adv Environ Eng Res

2021, volume 2, issue 2

doi:10.21926/aeer.2102008
Received: February 19, 2021

Accepted: April 18, 2021

Published: May 06,2021

\begin{abstract}
In this study, we used an image neural network model to assess the relationship between economic growth, pollution $\left(\mathrm{PM}_{2.5}, \mathrm{PM}_{10}\right.$, and $\left.\mathrm{CO}_{2}\right)$, and deaths from COVID-19 in the Hubei area (China). Data analysis, neural network analysis, and deep learning experiments were carried out to assess the relationship among COVID-19 deaths, air pollution, and economic growth in China (Hubei province, the epicenter of the COVID-19 pandemic). We collected daily data at a city level from January 20 to July 31, 2020. We used main cities in the Hubei province, with data on confirmed COVID-19 deaths, air pollution (expressed in $\mu \mathrm{g} / \mathrm{m}^{3}$ as $\mathrm{PM}_{2.5}, \mathrm{PM}_{10}$, and $\mathrm{CO}_{2}$ ), and per capita economic growth. Following the most recent contributions on the relationship among air pollution, GDP, and diffusion of COVID-19, we generated an algorithm capable of identifying a neural connection among these variables. The results confirmed a strong predictive relationship for the Hubei area between changes in the economic growth, fine particles, and deaths from COVID-19. These results would recommend adequate environmental reforms to policymakers to contain the spread and adverse effects of the virus. Therefore, there is a requirement to reconsider the system of transport and return to production by combining it with economic growth to protect the planet.
\end{abstract}

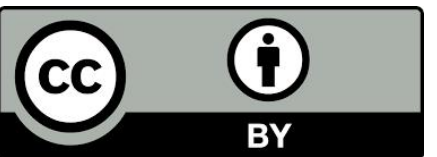

(c) 2021 by the author. This is an open access article distributed under the conditions of the Creative Commons by Attribution License, which permits unrestricted use, distribution, and reproduction in any medium or format, provided the original work is correctly cited. 


\section{Keywords}

Deep learning; air pollution; economic growth; COVID-19; China

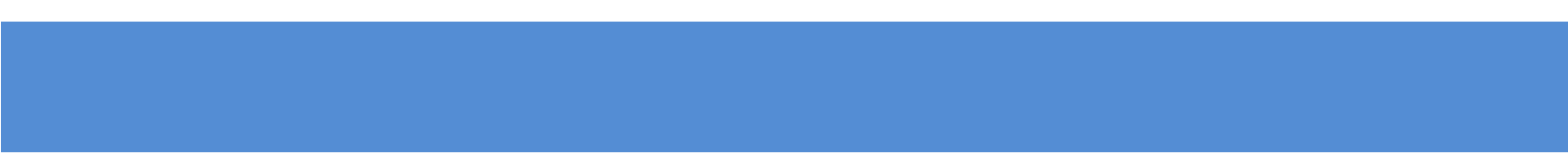

\section{Introduction}

Coronavirus 2019 (COVID-19) is a viral respiratory disease that can be contracted by being close to an infected person or with a contaminated surface. By early 2020 , the virus had spread to many countries in the world, forcing countries to begin practicing safety measures [1]. China was the epicenter of COVID-19 before it spread to other parts of the world in late 2019. In March 2020, the WHO declared COVID-19 as a global pandemic, paving the way for countries to take preventive measures. China had started dealing with the virus long before it was declared a global pandemic. The Asian countries began the practice of lockdown in their cities, controlling movements and grounding their planes. The Coronavirus pandemic situation slowed down China's economic growth, causing a major hit to the world's second-largest economy.

Doyle [2] reported that if the economic downturn continues for a year, then premature deaths related to air pollution could decrease by 50,000 to 100,000 , which is one of the few positive things about this crisis. It is important to understand its transmissibility and severity to judge how effectively the control measures in China have helped to stop the spread of the COVID-19 virus. This would help to gain information to control COVID-19 outbreaks in the world and its future potential re-emergence. In previous analyses of transmission dynamics in China, Kucharski et al. [3] fitted a stochastic transmission dynamic model to four datasets of cases from Wuhan and just considered the period up to February 11, 2020.

A wide range of studies that focused on air pollution and related deaths in China indicated that metropolitan cities in China contributed significantly to higher mortality rates in China as many developed respiratory-related issues due to exposure to polluted air. Although industrialization and urbanization are some of the significant economic growth factors in China and other parts of the world, they are also the primary causes of respiratory problems and deaths in major Chinese cities. Srivastava [4] offered a review on the intricate relationship between COVID-19, air pollution, and meteorology.

Several studies [5-7] analyzed the relationship among atmospheric stability/turbulence (considering wind speed), air pollution, and the spread of COVID-19 to provide insights into environmental risk factors of specific regions. Magazzino et al. [8] investigated the relationship between renewable energy and GDP acceleration in Brazil that could offset the harmful effects of the COVID-19 global pandemic. Magazzino et al. [9] assessed the relationship between COVID-19related deaths, economic growth, $\mathrm{PM}_{10}, \mathrm{PM}_{2.5}$, and $\mathrm{NO}_{2}$ concentrations in the New York state. Mele et al. [10] found that threshold values of $\mathrm{NO}_{2}$ connected to COVID-19 ranged from 15.8 $\mu \mathrm{g} / \mathrm{m}^{3}$ for Lyon, $21.08 \mu \mathrm{g} / \mathrm{m}^{3}$ for Marseille, and $22.9 \mu \mathrm{g} / \mathrm{m}^{3}$ for Paris. Shen et al. [11] studied the link between pollution events and lockdown periods in Hubei. Zhang et al. [12] applied a mathematical model with multiple datasets to estimate the transmissibility of the COVID-19 virus and the severity of the illness associated with the infection, and how both were affected by 
unprecedented control measures. Bashir et al. [13] employed the Spearman's and Kendall correlation tests to analyze the association of $\mathrm{PM}_{2.5}, \mathrm{PM}_{10}, \mathrm{SO}_{2}, \mathrm{NO}_{2}, \mathrm{~Pb}, \mathrm{VOC}$, and CO with COVID19 cases in California. Coccia [14] proposed the Index $c$ that quantifies the environmental risk of exposure of cities/regions to future epidemics of the COVID-19 and similar vital agents. Studies have $[15,16]$ explained the relationship between wind speed, air pollution, and the diffusion of COVID-19 to provide insights to constrain and/or prevent future pandemics and epidemics. Haque and Rahman [17] determined the association between average temperature and humidity with the COVID-19 pandemic in Bangladesh. Linear regression results showed that high temperature and high humidity significantly reduced the transmission of COVID-19. Mele and Magazzino [18] showed a unidirectional causal link between economic growth and $\mathrm{PM}_{2.5}, \mathrm{CO}_{2}$, and $\mathrm{NO}_{2}$. The D2C algorithm results highlighted a direct relationship between the concentration of $\mathrm{PM}_{2.5}$ and COVID19 deaths. Udemba et al. [19] explored the interactions among pollutant emission, foreign direct investment, energy consumption, tourism arrival, and economic growth in China for quarterly frequency data from 1995Q1 to 2016Q4. The empirical results showed a positive relationship between pollutant emissions and all other variables, except economic growth. Rosario et al. [20] evaluated the relationship between weather factors (temperature, humidity, solar radiation, wind speed, and rainfall) and COVID-19 infection in the State of Rio de Janeiro. Xie and Zhu [21] explored the nonlinear relationship between ambient temperature and COVID-19 infection in 122 cities from China. Xu et al. [22] inspected the air quality of three cities in the Hubei province to analyze the impact of the epidemic prevention and control actions on air quality.

In the present study, the neural network (NN) model was used to assess the relationship between economic growth, pollution $\left(\mathrm{PM}_{2.5}, \mathrm{PM}_{10}\right.$, and $\left.\mathrm{CO}_{2}\right)$, and deaths related to COVID-19 in China (in the Hubei area). The novelty aspects of the study concerned the application of the NN algorithm to investigate the intricate nexus among air pollution, economic growth, and COVID-19related deaths in the case of Hubei. Moreover, we adopted an extremely innovative empirical strategy, applying an imaging deep learning (DL) process.

\section{Materials and Methods}

To assess the relationship among COVID-19 deaths, air pollution, and economic growth in China (Hubei area), the daily data at a city level were collected from January 20 to July 31, 2020.

The present study focused on a case study of the Chinese province of Hubei, the first area in the world that experienced a rapid increase in the confirmed cases of COVID-19 and related deaths. The sources of data are the Chinese Center for Disease Control and Prevention (http://www.chinacdc.cn/) for numbers of infected people and deaths; the Hubei Environmental Protection Agency (http://sthjt.hubei.gov.cn/) for levels of air pollution; and the institutional website of the three main cities of Hubei province for per capita economic growth series (en.yichang.gov.cn; en.xiangyang.gov.cn; en.whuan.gov.cn).

To generate a daily time series relating to the variable of economic growth, we used the monthly data and standardized the data for the number of days. This procedure is suitable for the ML process. The machine interprets the data as an aggregate of data and not as a time series. Each variable was transformed into logarithms $(I n)$, first differences $(d)$, squared $(s)$, and logarithmic differences (d.In). Thus, we have a dataset with 8,100 observations. Several observations justify the selection of an experiment in DL. 
Following the protocols used by Gardner and Dorling [23], Magazzino et al. [24-26], and Mele and Magazzino [27], we performed the DL experiment where NNs work in parallel and are, therefore, can process large data simultaneously and autonomously.

The use of ANNs, as a subset of the ML tools, follows a precise implementation scheme for building the network and obtaining results.

Therefore, after uploading the dataset in Oryx, we have adapted the data series to an ML process through logarithmic and differential transformations. A higher volume of data leads to a greater capacity of the automatic intelligence system to conduct analysis. The values are subsequently normalized through the training selection procedure, which also begins a first test phase. In this phase, we evaluated the presence of omitted variables or outliers, which might result in false values of the NN. Subsequently, starting from the results obtained with the first test, we selected the suitable number of hidden neurons that will be an integral part of the network. The next step was to launch the algorithm for creating the NN. The result obtained was carefully analyzed; we verified the precision and generalization of the obtained network.

\section{Results and Discussion}

In the first experiment, we tested the predictive capacity of 20 inputs concerning 8,100 combinations on five targets. We adapted the NN algorithm to predict the probability that each variable might cause a variation between the same variables. The number of inputs was 20, whereas the number of outputs was 5 . The complexity, represented by the numbers of hidden neurons, is $12: 10: 8: 6: 12: 5$. The construction of the NN was assessed through two complex DL models.

The incremental order test hypothesizes the presence of numerous better alternative NN models than the one selected. In particular, this test defined several errors (testing and training) lower than the NN of our model. Thus, if there is a better model than the estimated one, the test showed a high level of errors in both training and testing. The second model was based on the Minkowski error concerning the Quasi-Newton method algorithm. This method computes an approximation of the inverse Hessian at each iteration of the algorithm by only using gradient information.

Both tests confirmed the goodness of the selected NN model. The incremental order test showed the absence of an alternative NN to ours. Furthermore, the Quasi-Newton method showed that the error in the transmission of the NN, with the growth of the gradients descendent, is gradually lower and tending toward zero.

Therefore, the results of the NN signal prediction were generated. This system represented a pure DL image model. In other words, simulating an NN, the model showed how the signals are interconnected with each other. This result represented a causal link between thousands of combinations, showing the effect toward one of the five targets without the operator's intervention.

It is observed that per capita GDP, $\mathrm{PM}_{2.5}, \mathrm{PM}_{10}$, and $\mathrm{CO}_{2}$ meet in a top-level neuron layer. This situation suggests the presence of interconnections between these variables. The joint signal of the inputs meets, after a higher neural level, the target Deaths. In other words, the COVID-19 deaths in Hubei were affected by the level of pollutants in the environment that would have favored the process of deaths in the area under study. Usually, a model of NN signaled by images 
does not highlight joint processes. Instead, no further analysis elements were generated in our case. We noted how the variation in per capita GDP meets the variation of fine particulates in an early neural stage. In this situation, $\mathrm{CO}_{2}$ is absent. The fine particulates, inevitably generated by the process of economic growth and greater use of energy, generate the variation of deaths from COVID-19. This result was significant. It can be concluded that the continuous emission of fine particles increased the probability of generating still deaths from viral pandemics in the future. To test the results obtained through a different model, we used the image optimization one of the Adam algorithms. In particular, we investigated whether future variations of COVID-19 deaths in the Hubei zone can be caused by a relationship between economic growth and pollution (Figure 1). 
a) Relationship between GDP and $\mathrm{PM}_{2.5}$

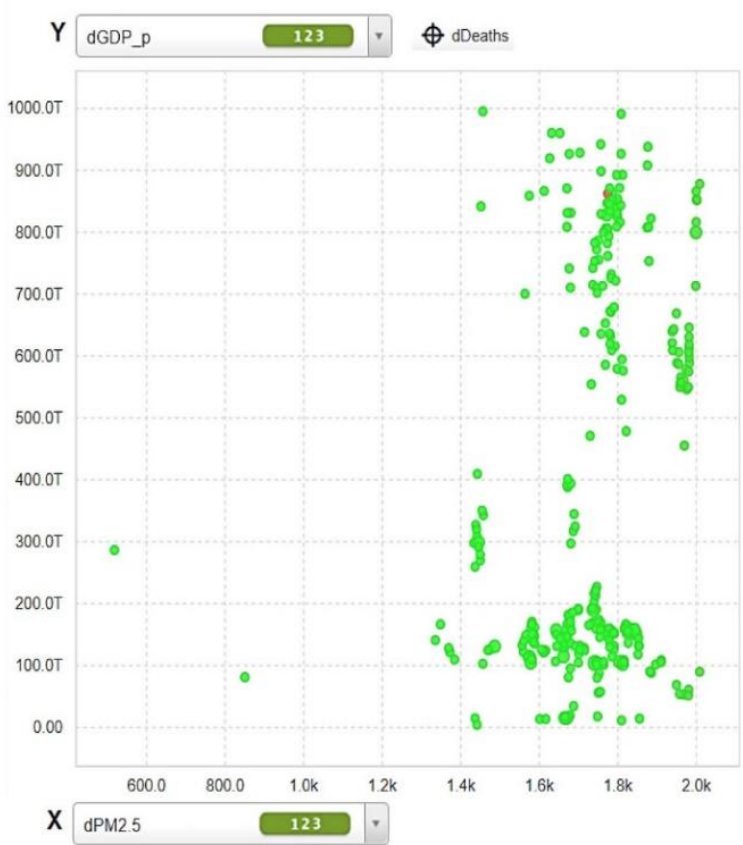

c) Relationship between GDP and $\mathrm{CO}_{2}$

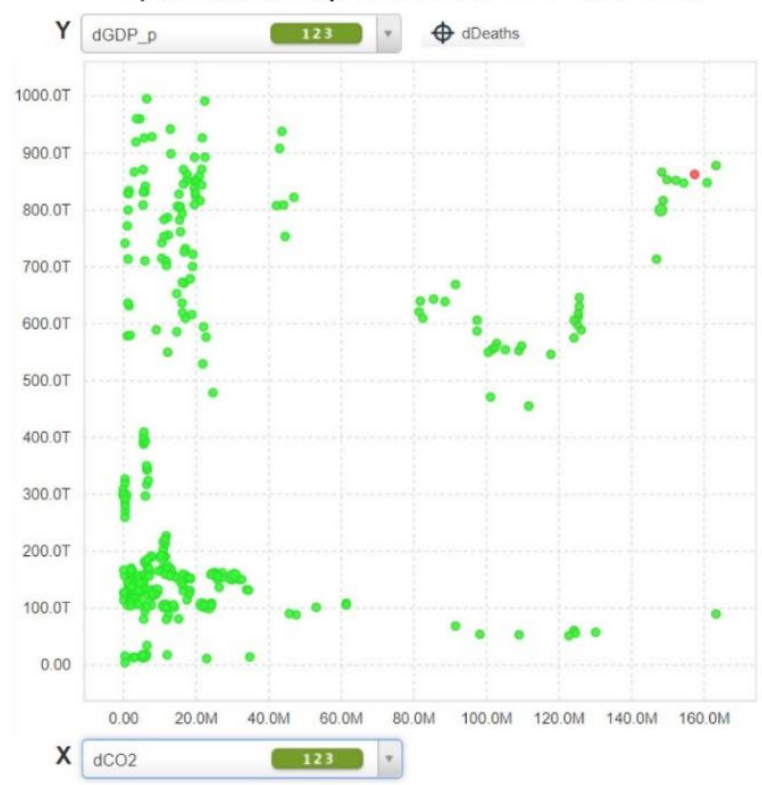

b) Relationship between GDP and $\mathrm{PM}_{10}$

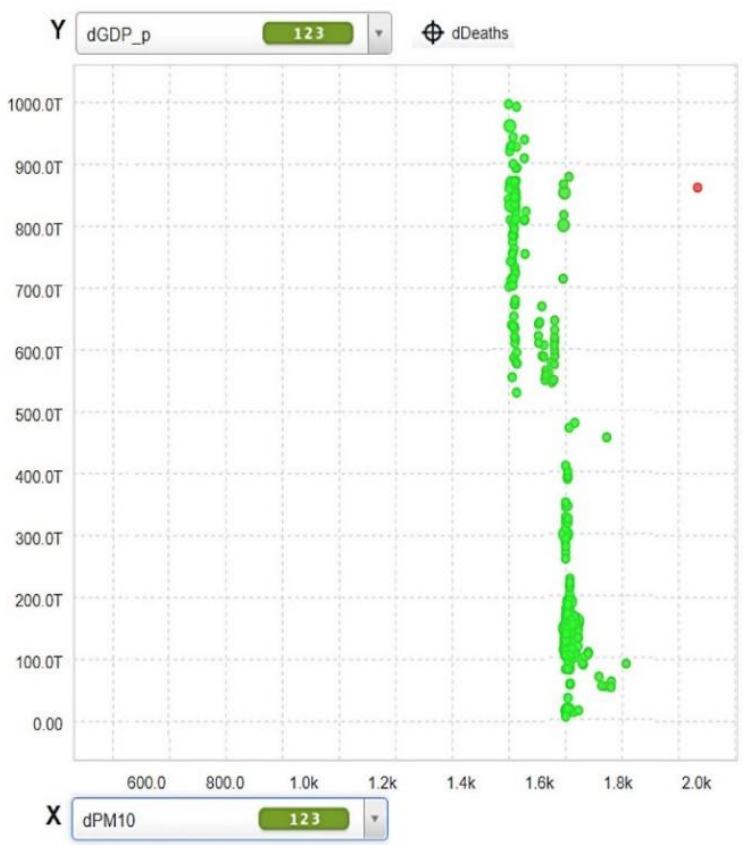

Notes: dGDP_p: GDP growth rate; $\mathrm{dPM}_{2.5}: \mathrm{PM}_{2.5}$ growth rate; $\mathrm{dPM}_{10}: \mathrm{PM}_{10}$ growth rate; $\mathrm{dCO}_{2}: \mathrm{dCO}_{2}$ growth rate.

Source: our elaborations in Oryx 2.0.8 and BML.

Figure 1 Imaging optimization: dGDP_p; $\mathrm{dPM}_{2.5} ; \mathrm{dPM}_{10} ; \mathrm{dCO}_{2}$.

As seen from Figure 1, the results partially confirmed those of the NN. We can see that, by targeting deaths, fine particulates $\left(\mathrm{PM}_{2.5}\right.$ and $\left.\mathrm{PM}_{10}\right)$ are closely linked to the change in economic growth. As per capita income grows, we noticed an increase in the variation of both $\mathrm{PM}_{2.5}$ and $\mathrm{PM}_{10}$ particulates, and in turn, these variables generated the variance of deaths. On the contrary, this predictive situation does not exist in the figure concerning $d \mathrm{CO}_{2}$. As per capita income 
increased, $\mathrm{CO}_{2}$ growth was evident only in the initial phase of the optimization process. This result underlined how pollutants are generated by economic growth.

To sum up, the DL results are in line with empirical findings presented in studies by Coccia [5], Magazzino et al. [9, 28], and Mele et al. [10] because a clear relationship emerged among air pollution, economic growth, and deaths due to COVID-19 pandemic.

Nevertheless, the hypothesis that the dust suspended in the atmosphere can transfer the virus inside the respiratory tract is a plausible situation but requires in-depth studies. We are convinced that it is a possible phenomenon but unlikely due to airborne viruses' concentration levels in outdoor environments. Mortality from COVID-19 is higher in individuals with previous copathologies such as pathologies linked to chronic exposure to atmospheric pollution, cardiovascular disease, hypertension, chronic breathing disorders, and cancer. Therefore, we can suggest a probable relationship between pollution and marked mortality of COVID-19 infection.

\section{Conclusion}

The COVID-19 pandemic has continued to spread worldwide since January 2020. Although the scientific research community is evaluating over 200 vaccine candidates to defeat the COVID-19 pandemic; however, there is currently no definitive cure. According to the conception proposed earlier by Becchetti et al. [29] and Dominici et al. [30] that fine particulates and carbon dioxide can play a direct and an indirect role in the diffusion and death from COVID-19, the present study focused its attention on the viral zone of Hubei. Interestingly, we have observed that NN has identified two pathways for the neural signal. First, from economic growth, $\mathrm{PM}_{2.5}$ and $\mathrm{PM}_{10}$ to the number of deaths due to COVID-19, and second, the variation in deaths could be caused by a variation (in acceleration) in economic growth and by pollutants. Finally, we have tested these latest results in an optimization model of the Adam algorithm. It showed a relationship between the change in economic growth and the change in fine particulates, which generate, in a target objective, variations in deaths due to COVID-19. These findings would confirm the hypothesis of a non-negligible connection between pollutants, economic growth, and a higher probability of spreading and death related to COVID-19 in a specific study area. There is, therefore, a need to reconsider the system of transport and return to production by combining it with growth economics to protect the planet.

Future developments might involve the application of the DL process with a larger set of variables, including meteorological factors (temperature, wind speed, humidity, etc.) to verify the previous results.

\section{Author Contributions}

Cosimo Magazzino: Conceptualization, Data curation, Formal analysis, Methodology, Software, Validation, Visualization, Writing - review\&editing.

Marco Mele: Conceptualization, Writing - original draft, Methodology, Software, Validation, Writing - original draft.

\section{Competing Interests}

The authors have declared that no competing interests exist. 


\section{References}

1. Khan N, Faisal S. Epidemiology of corona virus in the world and its effects on the China economy. SSRN. 2020. Doi: 10.2139/ssrn.3548292.

2. Jain A, Doyle DJ. Stages or phenotypes? A critical look at COVID-19 pathophysiology. Intensive Care Med. 2020; 46: 1494-1495.

3. Kucharski AJ, Russell TW, Diamond C, Liu Y, Edmunds J, Funk S, et al. Early dynamics of transmission and control of COVID-19: A mathematical modelling study. Lancet Infect Dis. 2020; 20: 553-558.

4. Srivastava A. COVID-19 and air pollution and meteorology-an intricate relationship: A review. Chemosphere. 2020; 263: 128297.

5. Coccia M. Effects of the spread of COVID-19 on public health of polluted cities: Results of the first wave for explaining the dejà vu in the second wave of COVID-19 pandemic and epidemics of future vital agents. Environ Sci Pollut Res. 2021; 28: 19147-19154.

6. Coccia M. The effects of atmospheric stability with low wind speed and of air pollution on the accelerated transmission dynamics of COVID-19. Int J Environ Stud. 2021; 78: 1-27.

7. Coccia M. The relation between length of lockdown, numbers of infected people and deaths of Covid-19, and economic growth of countries: Lessons learned to cope with future pandemics similar to COVID-19 and to constrain the deterioration of economic system. Sci Total Environ. 2021; 775: 145801.

8. Magazzino $\mathrm{C}$, Mele $\mathrm{M}$, Morelli $\mathrm{G}$. The relationship between renewable energy and economic growth in a time of COVID-19: A machine learning experiment on the Brazilian economy. Sustainability. 2021; 13: 1285.

9. Magazzino C, Mele M, Sarkodie SA. The nexus between COVID-19 deaths, air pollution and economic growth in New York state: Evidence from deep machine learning. J Environ Manage. 2021; 286: 112241.

10. Mele M, Magazzino C, Schneider N, Strezov V. $\mathrm{NO}_{2}$ levels as a contributing factor to COVID-19 deaths: The first empirical estimate of threshold values. Environ Res. 2021; 194: 110663.

11. Shen L, Zhao T, Wang H, Liu J, Bai Y, Kong S, et al. Importance of meteorology in air pollution events during the city lockdown for COVID-19 in Hubei Province, Central China. Sci Total Environ. 2021; 754: 142227.

12. Zhang XS, Vynnycky E, Charlett A, De Angelis D, Chen Z, Liu W. Transmission dynamics and control measures of COVID-19 outbreak in China: A modelling study. Sci Rep. 2021; 11: 2652.

13. Bashir MF, Jiang $B$, Komal $B$, Bashir MA, Farooq $T H$, Iqbal $N$, et al. Correlation between environmental pollution indicators and COVID-19 pandemic: A brief study in Californian context. Environ Res. 2020; 187: 109652.

14. Coccia M. An index to quantify environmental risk of exposure to future epidemics of the COVID-19 and similar viral agents: Theory and practice. Environ Res. 2020; 191: 110155.

15. Coccia M. How do low wind speeds and high levels of air pollution support the spread of COVID-19? Atmospheric Pollut Res. 2021; 12: 437-445.

16. Coccia M. How (Un) sustainable environments are related to the diffusion of COVID-19: The relation between coronavirus disease 2019, air pollution, wind resource and energy. Sustainability. 2020; 12: 9709.

17. Haque SE, Rahman M. Association between temperature, humidity, and COVID-19 outbreaks 
in Bangladesh. Environ Sci Policy. 2020; 114: 253-255.

18. Mele M, Magazzino C. Pollution, economic growth, and COVID-19 deaths in India: A machine learning evidence. Environ Sci Pollut Res. 2021; 28: 2669-2677.

19. Magazzino C, Udemba EN, Bekun F. Modelling the nexus between pollutant emission, energy consumption, foreign direct investment and economic growth: New insights from China. Environ Sci Pollut Res. 2020; 27: 17831-17842.

20. Rosario DK, Mutz YS, Bernardes PC, Conte-Junior CA. Relationship between COVID-19 and weather: Case study in a tropical country. Int J Hyg Environ Health. 2020; 229: 113587.

21. Xie J, Zhu Y. Association between ambient temperature and COVID-19 infection in 122 cities from China. Sci Total Environ. 2020; 724: 138201.

22. Xu K, Cui K, Young LH, Hsieh YK, Wang YF, Zhang J, et al. Impact of the COVID-19 event on air quality in central China. Aerosol Air Qual Res. 2020; 20: 915-929.

23. Gardner MW, Dorling SR. Artificial neural networks (the multilayer perceptron)-a review of applications in the atmospheric sciences. Atmospheric Environ. 1998; 32: 2627-2636.

24. Magazzino C, Mele M, Schneider N. A D2C algorithm on the natural gas consumption and economic growth: Challenges faced by Germany and Japan. Energy. 2021; 219: 119586.

25. Magazzino C, Mele M, Schneider N. A machine learning approach on the relationship among solar and wind energy production, coal consumption, GDP, and $\mathrm{CO}_{2}$ emissions. Renew Energ. 2021; 167: 99-115.

26. Magazzino $C$, Mele $M$, Schneider N, Shahbaz M. Can biomass energy curtail environmental pollution? A quantum model approach to Germany. J Environ Manage. 2021; 287: 112293.

27. Mele $M$, Magazzino $C$. A machine learning analysis of the relationship among iron and steel industries, air pollution, and economic growth in China. J Clean Prod. 2020; 277: 123293.

28. Magazzino C, Mele M, Schneider N. The relationship between air pollution and COVID-19related deaths: An application to three French cities. Appl Energy. 2020; 279: 115835.

29. Becchetti L, Conzo G, Conzo P, Salustri F. Understanding the heterogeneity of adverse COVID19 outcomes: The role of poor quality of air and lockdown decisions. SSRN. 2020. Doi: $10.2139 /$ ssrn.3572548.

30. Wu X, Nethery RC, Sabath BM, Braun D, Dominici F. Exposure to air pollution and COVID-19 mortality in the United States. MedRxiv. 2020. Doi: 10.1101/2020.04.05.20054502.
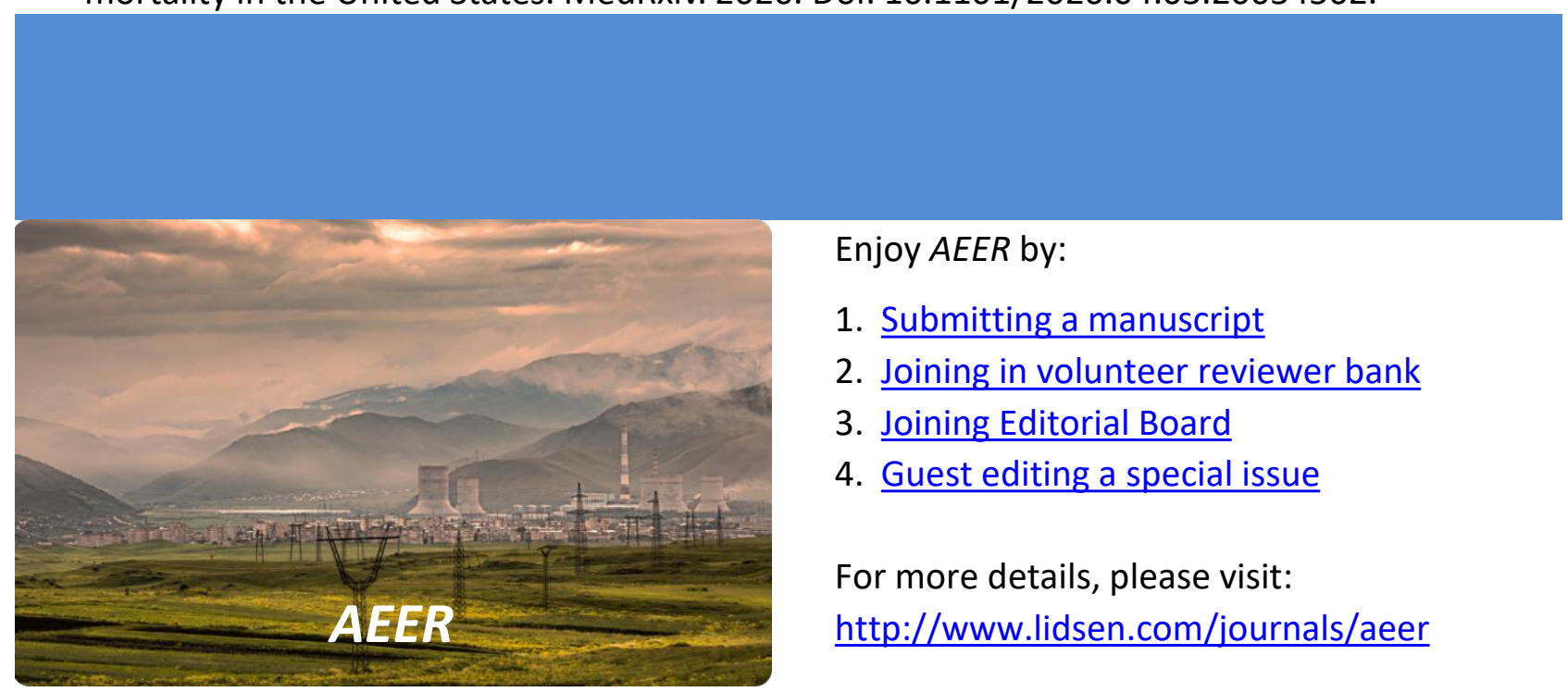

Enjoy AEER by:

1. Submitting a manuscript

2. Joining in volunteer reviewer bank

3. Joining Editorial Board

4. Guest editing a special issue

For more details, please visit: http://www.lidsen.com/journals/aeer 
Adv Environ Eng Res 2021; 2(1), doi:10.21926/aeer.2102008 